\title{
Logit and Fuzzy Models in Data Analysis: Estimation of Risk in Cardiac Patients
}

\author{
P. HONZÍK ${ }^{1}$, L. KŘIVAN ${ }^{2}$, P. LOKAJ $^{2}$, R. LÁBROVÁ ${ }^{2}$, Z. NOVÁKOVÁ ${ }^{3}$, B. FIŠER ${ }^{3}$, \\ N. HONZÍKOVÁ ${ }^{3}$
}

${ }^{1}$ Department of Control and Instrumentation, Brno University of Technology, Brno, Czech

Republic, ${ }^{2}$ Department of Internal Cardiology and ${ }^{3}$ Department of Physiology, Faculty of Medicine, Masaryk University, Brno, Czech Republic

Received February 16, 2010

Accepted March 26, 2010

\section{Summary}

The aim of this study was a comparison of risk stratification for death in patients after myocardial infarction (MI) and of risk stratification for malignant arrhythmias in patients with implantable cardioverter-defibrillator (ICD). The individual risk factors and more complex approaches were used, which take into account that a borderline between a risky and non-risky value of each predictor is not clear-cut (fuzzification of a critical value) and that individual risk factors have different weight (area under receiver operating curve - AUC or Sommers' D - Dxy). The risk factors were baroreflex sensitivity, ejection fraction and the number of ventricular premature complexes/hour on Holter monitoring. Those factors were evaluated separately and they were involved into logit model and fuzzy models (Fuzzy, FuzzyAUC, and Fuzzy-Dxy). Two groups of patients were examined: a) 308 patients 7-21 days after MI (23 patients died within period of 24 month); b) 53 patients with left ventricular dysfunction examined before implantation of ICD (7 patients with malignant arrhythmia and electric discharge within 11 month after implantation). Our results obtained in MI patients demonstrated that the application of logit and fuzzy models was superior over the risk stratification based on algorithm where the decision making is dependent on one parameter. In patients with implanted defibrillator only logit method yielded statistically significant result, but its reliability was doubtful because all other tests were statistically insignificant. We recommend evaluating the data not only by tests based on logit model but also by tests based on fuzzy models.

\section{Key words}

Risk prediction • Myocardial infarction • Implantable cardioverterdefibrillator • Fuzzy logic • Area under receiver operating characteristic $\bullet$ Logistic regression

\section{Corresponding author}

N. Honzíková, Department of Physiology, Faculty of Medicine, Masaryk University, Komenského nám. 2, CZ-662 43 Brno, Czech Republic.

Phone/FAX: +420-549 493 748. E-mail: nhonziko@med.muni.cz

\section{Introduction}

Prediction of the risk of cardiac death based on determination of critical values of non-invasive risk factors was first studied in patients after myocardial infarction. The increased risk of cardiac death is caused by an ischemia-induced non-uniform conduction velocity in different myocardial cells, e.g. an arrhythmogenic terrain; an increased automaticity of ventricular myocytes, e.g. a triggering factor; an increased sympathetic and/or decreased parasympathetic autonomic nervous activity, which decreases the gain of autonomous reflexes protecting the heart; and a decreased contractility of myocardium leading to heart failure (Mortara et al. 1996, La Rovere et al. 1998, Honzíková et al. 2000a, Bailey et al. 2007, Greiser et al. 2009). The studies of risk predictors of cardiac death were essential for the decision to implant a cardioverter defibrillator after MI (Moss et al. 1996, Bailey et al. 2007).

Generally, identification of risk predictors is 
based on determination of their sensitivity, specificity, and positive predictive value (PPV) at a critical value determined by calculating receiver operating curve ROC (Camm and Fei 1995, Honzíková et al. 2000b, Krontorádová et al. 2008), for a group of risk factors by the logistic regression analysis and calculation of odds ratio (Mangoni et al. 2003). The improvement of a predictive power of a group of risk factors may be reached taking into account different weight of predictors and also the physiological assumption that the edge between a risky and non-risky value of each predictor is not clear-cut using fuzzification of a critical value (Honzík et al. 2003, Honzíková et al. 2003).

Further requirements are associated with the strategy of determination of a risk of patient with respect to the therapeutic strategy. For example, high sensitivity at PPV of 0.5 is desirable in patients in which it is necessary to avoid an unnecessary treatment (Camm and Fei 1995).

The aim of this study was further development of fuzzified and weighted models for risk stratification and comparison of the predicting power of standard individual predictors and these new models in two groups of patients. A number of risk factors for cardiac death in patients after MI were evaluated: the presence of ventricular premature complexes representing arrhythmogenic terrain, baroreflex sensitivity representing decreased parasympathetic activity, and ejection fraction representing decreased contractility. The same predictors were evaluated as risk factors for the occurrence of malignant arrhythmia in patients with implantable defibrillator.

\section{Methods}

\section{Participants and study design}

Two groups of patients were examined. The first one consisted of 308 patients 7-21 days after MI (aged $57.5 \pm 8.8$ years) including 23 patients who died (cardiac death) in a course of the two-years' follow-up period (aged 63.3 \pm 6.1 years). These subjects were included into statistics as risky patients. The second group included 53 patients (aged $60.0 \pm 12.4$ years), with left ventricular dysfunction (LVEF $\leq 40 \%$ ) of mixed aetiology (38 with ischemic heart disease, 15 with dilatation cardiomyopathy). These patients were examined before implantation of cardioverter-defibrillator (ICD). Noninvasive examination was carried-out 6 weeks after MI in group with coronary artery disease. LVEF increased in some patients after the angioplasty within 6 weeks. Furthermore, some patients underwent programmed ventricular pacing by electrophysiological study and examination of microvolt $\mathrm{T}$ wave alternans (MTWA) during bicycle ergometry. After ICD implantation, the information about malignant arrhythmias was obtained from the ICD memory by regular ambulatory check-up. Seven patients of the second group (aged 55.9 \pm 7.3 years) with documented cardiac arrest or with ventricular arrhythmias faster as 250/min within 11 month after implantation of ICD were included into statistics as risky patients. In both groups of patients, ejection fraction (EF in \%), ventricular premature complexes (VPCs/h in number/hour) and baroreflex sensitivity (BRS in $\mathrm{ms} / \mathrm{mmHg}$ ) were examined and evaluated as predictors of risk of patients for cardiac death after MI or malignant arrhythmias in patients with ICD.

The Ethics Committee of Masaryk University approved of this study and all patients gave their informed consent.

\section{Ejection fraction}

Two-dimensional echocardiogram was obtained using the Acuson $128 \mathrm{XP} / 10$ unit in the first group patients (after MI) and a Vivid 7 GE Medical Systems Milwaukee, VVI in the second group (ICD patients). The left ventricular ejection fraction was evaluated.

\section{Holter monitoring}

A two-channel, 24-hour ECG recording (Oxford Excell) was performed. The recordings were manually edited, the artefacts were discarded. Arrhythmias ware evaluated and classified (ventricular premature complexes as simple, bigeminal, multiform, repetitive or $\mathrm{R}$ on $\mathrm{T}$ ), and the count of ventricular ectopic beats per hour (VPCs/h) was determined.

\section{Baroreflex sensitivity determination by spectral method}

Indirect continuous 5-minute blood pressure recordings from finger arteries (Finapres, Ohmeda, Madison, USA) were performed in sitting, resting subjects. The recordings were taken during controlled breathing at 20 breaths per min by metronome $(0.33 \mathrm{~Hz})$; the subjects were allowed to adjust the tidal volume according to their own comfort.

Beat-to-beat values of systolic blood pressure and of inter-beat intervals were measured for further analysis. For the spectral analysis, the parameters were linearly interpolated and equidistantly sampled at $2 \mathrm{~Hz}$. 
The linear trend was removed. The autocorrelation and cross-correlation functions, power spectra and crossspectra, coherence and the modulus between inter-beat intervals and systolic blood pressure were calculated. The gain factor, i.e. the transfer function among variations in systolic blood pressure and inter-beat intervals, was calculated and its value at a frequency of $0.1 \mathrm{~Hz}$ was taken as a measure of BRS (Honzíková et al. 1992, Závodná et al. 2006).

\section{Statistical analysis}

For the prediction of the binary output 4 following approaches were applied: logistic regression (Logit), fuzzy model (Fuzzy) and two fuzzy weighted models (Fuzzy-AUC, Fuzzy-Dxy).

Logistic regression is used to predict the probability of the binary output variable. Its output value confines between 0 and 1 . The logistic model consists of the logistic function $1 /(1+\exp (-z))$ and the linear function $\mathrm{z}=\mathrm{b}_{0}+\mathrm{b}_{1} * \mathrm{x}_{1}+\mathrm{b}_{2} * \mathrm{x}_{2}+\ldots$ (in our study $\mathrm{x}_{1}=\mathrm{BRS}[\mathrm{ms} / \mathrm{mmHg}$; $\mathrm{x}_{2}=\mathrm{EF}[\%] ; \mathrm{x}_{3}=\mathrm{VPCs}$ [number/hour]). The regression coefficients $b_{i}$ are calculated with the maximum likelihood estimation (Frank et al. 2001).

The fuzzy model takes into account that the edge between a risky and non-risky value of each predictor is not distinct. The measure of the increasing/decreasing risk of each single predictor is determined with one fuzzy set with the output range from 0 to 1 . The final measure of risk is determined as the sum of partial risks of individual predictors. The value of the measure is in the range from 0 to the count of predictors (3 in our case BRS, EF, and VPCs). Piecewise linear membership functions were used for fuzzification of the individual predictors. The fuzzification of the individual predictors was described previously (Honzik et al. 2003).

Fuzzy weighted method Fuzzy-AUC multiplies each individual risk of each predictor with the predictor's area under receiver operating characteristic (AUC). The AUC is the area under the plot of sensitivity versus specificity for moving critical values in steps (Bradley et al. 1997).

Next, the sum of weighted risks is computed. The output value is than in the range from 0 to sum of AUCs of all individual predictors.

The disadvantage of AUC in the role of weight is its range from 0 to 1 , where 0.5 is equivalent to a random variable. For this reason the Somers' Dxy Rank Correlation (Dxy) was also used as the weight. Dxy is a linear transformation of AUC (Dxy=2*AUC-1) and its value ranges from -1 to 1 (Frank et al. 2001). For this reason it is more suitable as a weighting factor.

To compare the quality of prediction, the measure of performance must be the same for all models. We used two measures which do not need determination of the critical value: Wilcoxon rank-sum test and AUC. Furthermore we used sensitivity and specificity at a critical value. The critical value was determined: 1 . at the value of the model output where the maximal sum of sensitivity and specificity was achieved, and 2 . at the positive predictive value of 0.5 .

The prediction quality of the 4 tested models is compared with the prediction quality of the individual predictors.

\section{Results}

Comparison of predicting quality of all predictors in patients after $M I$

Comparison of EF, VPCs/h, and BRS shows that patients after $\mathrm{MI}$ have significantly lower EF, higher VPCs/h, and lower BRS compared with survivors (Table 1).

Table 1. Differences between survivors and deceased patients 24 month after myocardial infarction.

\begin{tabular}{lccc}
\hline Parameter & $\begin{array}{c}\text { Survivors } \\
\mathbf{( n = 2 8 5 )}\end{array}$ & $\begin{array}{c}\text { Deceased patients } \\
(\mathbf{n}=\mathbf{2 3})\end{array}$ & $\begin{array}{c}\text { P value } \\
\text { (Wilcoxon test) }\end{array}$ \\
\hline Ejection fraction (\%) & $47.7 \pm 9.5$ & $38.3 \pm 10.8$ & $\mathrm{p}=4 \cdot 10^{-5}$ \\
Ventricular premature complexes (number/h) & $7.73 \pm 29.1$ & $20.8 \pm 35.2$ & $\mathrm{p}=4 \cdot 10^{-4}$ \\
Baroreflex sensitivity (ms/mmH) & $5.48 \pm 4.53$ & $3.27 \pm 3.73$ & $\mathrm{p}=6 \cdot 10^{-4}$ \\
Logit & $0.07 \pm 0.08$ & $0.18 \pm 0.13$ & $\mathrm{p}=8 \cdot 10^{-6}$ \\
Fuzzy & $1.18 \pm 0.37$ & $1.65 \pm 0.43$ & $\mathrm{p}=9 \cdot 10^{-7}$ \\
Fuzzy-AUC & $0.86 \pm 0.27$ & $1.20 \pm 0.32$ & $\mathrm{p}=1 \cdot 10^{-6}$ \\
Fuzzy-Dxy & $0.54 \pm 0.17$ & $0.76 \pm 0.20$ & $\mathrm{p}=1 \cdot 10^{-6}$ \\
\hline
\end{tabular}


Table 2. Sensitivity, specificity, positive predictive value (PPV) for optimal critical values (o.c.v.), area under receiver operating curve (AUC), and sensitivity and specificity for PPV 0.5 in patients after myocardial infarction.

\begin{tabular}{lccccccc}
\hline Predictor & o.c.v. & Sensitivity & Specificity & PPV & AUC & $\begin{array}{c}\text { PPV }=\mathbf{0 . 5} \\
\text { Specificity }\end{array}$ \\
\hline BRS & 3.08 & 0.70 & 0.68 & 0.15 & 0.71 & 0.04 & 1 \\
VPCs $/ h$ & 1.21 & 0.65 & 0.70 & 0.15 & 0.72 & - & - \\
EF & 42.0 & 0.78 & 0.74 & 0.19 & 0.76 & 0.09 & 0.99 \\
Logit & 0.09 & 0.74 & 0.80 & 0.22 & 0.78 & 0.26 & 0.98 \\
Fuzzy & 1.46 & 0.78 & 0.82 & 0.26 & 0.81 & 0.09 & 0.99 \\
Fuzzy-AUC & 1.05 & 0.78 & 0.81 & 0.24 & 0.81 & 0.09 & 0.99 \\
Fuzzy-Dxy & 0.63 & 0.83 & 0.74 & 0.20 & 0.80 & 0.22 & 0.98 \\
\hline
\end{tabular}

Table 3. Differences between ICD patients (pts) with and without malignant arrhythmia (MA) 11 month after implantation of defibrillator.

\begin{tabular}{lccc}
\hline Parameter & $\begin{array}{c}\text { ICD pts } \\
\text { without } \mathbf{M A} \\
(\mathbf{n = 4 6 )}\end{array}$ & $\begin{array}{c}\text { ICD pts } \\
\text { with MA } \\
(\mathbf{n}=\mathbf{7})\end{array}$ & $\begin{array}{c}\text { P value } \\
\text { (Wilcoxon test) }\end{array}$ \\
\hline Ejection fraction (\%) & $30.5 \pm 8.1$ & $35.0 \pm 11.9$ & $\mathrm{~ns}(\mathrm{p}=0.26)$ \\
VPCs/h & $55.0 \pm 162$ & $387 \pm 615$ & $\mathrm{~ns}(\mathrm{p}=0.22)$ \\
Baroreflex sensitivity $(\mathrm{ms} / \mathrm{mmHg})$ & $6.97 \pm 6.18$ & $5.73 \pm 4.55$ & $\mathrm{~ns}(\mathrm{p}=0.82)$ \\
Logit & $0.10 \pm 0.10$ & $0.33 \pm 0.29$ & $\mathrm{p}<0.05$ \\
Fuzzy & $1.42 \pm 0.38$ & $1.59 \pm 0.82$ & $\mathrm{~ns}(\mathrm{p}=0.80)$ \\
Fuzzy-AUC & $0.83 \pm 0.22$ & $0.94 \pm 0.51$ & $\mathrm{~ns}(\mathrm{p}=0.86)$ \\
Fuzzy-Dxy & $0.23 \pm 0.06$ & $0.28 \pm 0.19$ & $\mathrm{~ns}(\mathrm{p}=0.92)$ \\
\hline
\end{tabular}

Table 4. Sensitivity, specificity, positive predictive value (PPV) for optimal critical values (o.c.v.), area under receiver operating curve (AUC), and sensitivity and specificity for PPV 0.5 in ICD patients (pts) with and without malignant arrhythmia 11 month after implantation of defibrillator.

\begin{tabular}{lccccccc}
\hline Predictor & o.c.v. & Sensitivity & Specificity & PPV & AUC & \multicolumn{2}{c}{ PPV $=\mathbf{0 . 5}$} \\
& & & & & & Sensitivity & Specificity \\
\hline BRS & 4.42 & 0.57 & 0.55 & 0.16 & 0.53 & - & 0.96 \\
VPCS/24h & 57.0 & 0.57 & 0.81 & 0.31 & 0.64 & 0.29 & 0.96 \\
EF & 34.0 & 0.71 & 0.51 & 0.18 & 0.63 & 0.29 & 0.92 \\
Logit & 0.12 & 0.71 & 0.72 & 0.28 & 0.74 & 0.57 & 0.96 \\
Fuzzy & 1.73 & 0.43 & 0.83 & 0.27 & 0.53 & 0.29 & 0.96 \\
Fuzzy-AUC & 1.02 & 0.43 & 0.83 & 0.27 & 0.52 & 0.27 & 0.96 \\
Fuzzy-Dxy & 0.31 & 0.43 & 0.89 & 0.38 & 0.51 & 0.29 & \\
\hline
\end{tabular}


Multiple logistic regression analysis reveals that low EF $(p<0.0007)$ is associated with increased risk of cardiac death after MI:

$$
\begin{aligned}
& \mathrm{z}=1.777632-0.153317 * \mathrm{BRS}- \\
& \quad-0.086452 * \mathrm{EF}+0.007173 * \operatorname{VPCs} \quad(\mathrm{p}<0.00002)
\end{aligned}
$$

For

Survivors $=0$

Deceased patients $=1$

ROC curves of all individual and new predictors (Logit, Fuzzy, Fuzzy-AUC, and Fuzzy-Dxy) are calculated. ROC curves of EF, VPCs/h, BRS, Logit and Fuzzy-Dxy are shown in Fig. 1. The shift of the curves of the new predictors to the right, e.g. an increase of their AUC, documents their higher predictive weight. The values of sensitivity, specificity, positive predictive value for optimal critical values, and AUC of all predictors calculated using ROC curves are presented in Table 2. Also sensitivity and specificity for PPV 0.5 is calculated (Table 2). Logit, Fuzzy, Fuzzy-AUC, and Fuzzy-Dxy models have higher predicting power compared with individual predictors.

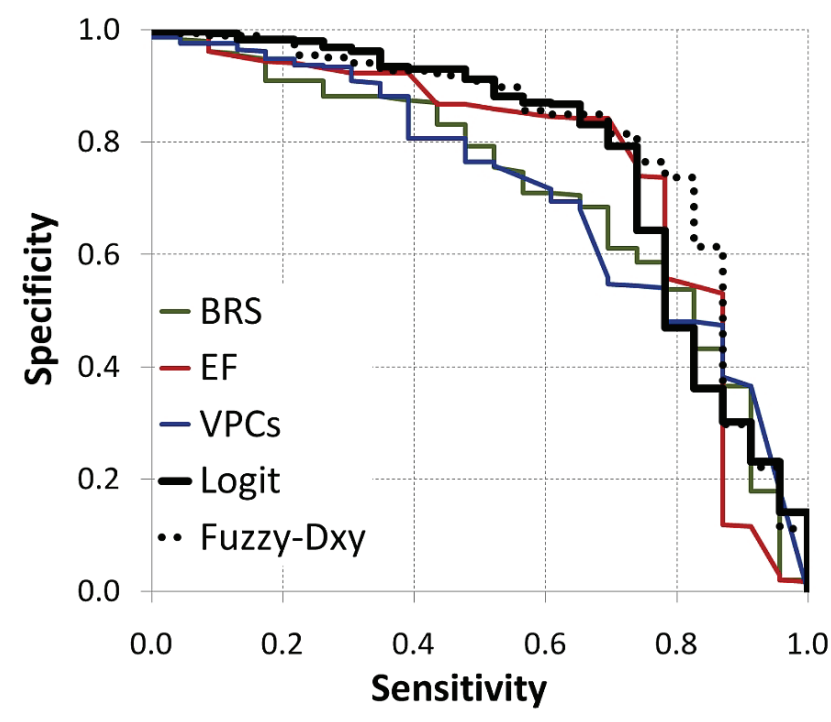

Fig. 1. Receiver operating curves of baroreflex sensitivity (full green line), ventricular premature complexes (full blue line), ejection fraction (full red line), Logit (full black line), and FuzzyDxy (black doted line) in patients after myocardial infarction who are at risk for cardiac death.

Comparison of predicting quality of all predictors in patients with ICD

Comparison of the predicting power of individual predictors and Logit, Fuzzy, Fuzzy-AUC, and
Fuzzy-Dxy models are summarized in Table 3; ROC curves are in Fig. 2; the values of sensitivity, specificity, positive predictive value for optimal critical values, AUC, and sensitivity and specificity for PPV 0.5 are in Table 4.

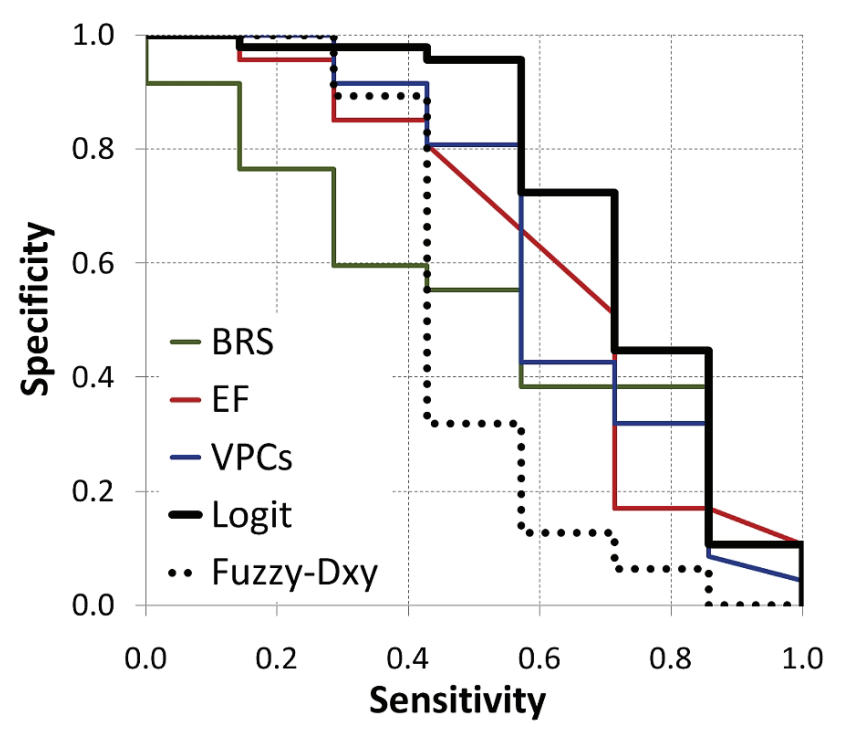

Fig. 2. Receiver operating curves of baroreflex sensitivity (green line), ventricular premature complexes (blue line), ejection fraction (red line), Logit (thick full line), and Fuzzy-Dxy (thick doted line) in ICD patients who are at risk for malignant arrhythmias.

Multiple logistic regression analysis revealed that increased presence of VPCS $(p<0.05)$ was associated with increased occurrence of malignant arrhythmias in patients with implantable defibrillator but the logit model itself was insignificant (chi-square test):

$$
\begin{aligned}
\mathrm{z}=-4.8968-0.054247 * \mathrm{BRS} & +0.086918^{*} \mathrm{EF}+ \\
& +0.00011562 * \operatorname{VPCS} \text { (n.s.) }
\end{aligned}
$$

For

ICD patients without $\mathrm{MA}=0$

ICD patients with $\mathrm{MA}=1$

Despite the logit model is not statistically significant, the AUC value of the logit model increased from 0.64 (VPCS) to 0.74 and the measure of separability expressed by the Wilcoxon test becomes statistically significant (Table 3).

\section{Discussion}

Two different approaches to modelling the relations in the data are presented in this study: the logistic regression and fuzzy modelling. The former 
model describes the relation between binary dependent variable and the set of independent variables. If there is a new independent variable added to the existing data, the newly assessed logistic model is larger with one regression coefficient. Moreover, the original regression coefficients change due to combined interaction between the new variable and the original set of variables. Thus, the logistic model cannot be additionally extended with a new variable without change of all original coefficients (Frank et al. 2001).

The logistic regression is a strictly statistical method which does not take into account any a priori knowledge. This is one of the reasons, why it is used i.a. for data analysis and for revealing the new relations among the predictors and the output attribute. In case of small data-sets or data-sets with strongly screwed class frequencies (only small percentage of positive outputs) the single predictors are often insignificant and the interpretation of the final logit model is not clear. The methods and approaches for the logit model evaluation can lead to different interpretations in such cases.

The advantages of the suggested fuzzy models over the logistic regression are their intuitive interpretation and additionality which means that the models can be extended with the new variable without any need to recompute the already known coefficients (fuzzifications).

The fuzzy modelling is based firstly on the a priori knowledge of the experts and secondly on the used datasets. The qualitative setup of fuzzy sets (orientation of membership functions) corresponds to the already known facts and can be simply interpreted. The quantitative setup of the fuzzy sets can be computed from the modelled data. There are more approaches leading to different setup of the fuzzy sets which influence the output results. On the other hand the different quantitative setups never lead to qualitatively different interpretations. The setup of fuzzy models can even include the information about generally accepted critical values or can be extended with weights (Fuzzy-Dxy) etc. (Buckley et al. 2002).

Our results in the MI patients demonstrate that the application of logit and fuzzy models is superior over the risk stratification based on an algorithm where the decision making depends on one parameter only. The most important parameter in estimation of the risk of death in MI patients is EF; BRS is slightly less and VPCs/h are markedly less important. The sensitivity and specificity of the test based on logit or fuzzy models are better. It is not surprising because it confirms our previous study of MI patients where we demonstrated that the number of risk factors (EF under $40 \%$, BRS under $3 \mathrm{~ms} / \mathrm{mmHg}$ and/or low heart rate variability, occurrence of late potentials, more than 10 VPCs per hour on Holter monitoring) is essential for risk stratification (Honzíková et al. 2000a). Similar data were found in other laboratories (La Rovere et al. 1998, Bailey et al. 2007). Such approach uses given threshold values as $40 \%$ for $\mathrm{EF}, 3 \mathrm{~ms} / \mathrm{mmHg}$ for BRS and the frequency of VPCs/h. We have exactly determined critical value for BRS calculated by non-invasive spectral method (Honzikova et al. 2000b) which was previously known only for phenylephrine method (La Rovere et al. 1998).

In analysis of ICD patients' data brings out a different situation. The most important factor for discharge trigger is the number of VPCs/h. BRS and EF are less important. The logit method yields statistically significant result (see Table 3), but the reliability of such a result is ambiguous because all other tests are statistically insignificant. Moreover, high EF in the logit equation is a risk factor which is in contradiction to all other sudden cardiac death studies. Thus, insignificance of the tests based on the fuzzy models seems to be correct. Indication for ICD implantation on the basis of VPCs/h, BRS and EF measurement cannot be further improved. Our results also indicate that to rely on one test, in our case the logit model, may be misleading. The recommendation to evaluate the data not only by a test based on logit model but also by tests based on fuzzy models is justified.

In some respect, the advantage of logit and/or fuzzy models is that the need for critical values is eliminated. From this point of view, the fuzzy model is superior over the logit model, because it enables also the inclusion of some a priori knowledge, e.g. critical values (Honzik et al. 2003). Another advantage of fuzzy model is the possibility to extend it with a new predictor without a need to recalculate the already used fuzzy sets of predictors.

The present method offers advancement in risk stratification based on inclusion of several weighted and fuzzified risk factors in one individual predictor. Such predictor can be used for binary discrimination between risky and non risky patients as for example in the decision process for implantation of defibrillator in patients with decreased EF of different aetiology.

In case that the logistic regression results are partially insignificant, the additional information provided by the fuzzy model can help to strengthen the significance of such logit model (our group of patients 
after $\mathrm{MI}$ ) or reject the potentially interesting relations revealed in the data (ICD patients).

\section{Conflict of Interest}

There is no conflict of interest.

\section{Acknowledgements}

Supported by grants: MSM 0021622402 from the Ministry of Education, Youth and Sports of the Czech Republic, MZ NS10421-3 and GA102/09/1897.

\section{References}

BAILEY JJ, HODGES M, CHURCH TR: Decision to implant a cardioverter defibrillator after myocardial infarction: The role of ejection fraction v. other risk factor markers. Medical Decision Making 27: 151-160, 2007.

BRADLEY AP: The use of the area under the ROC curve in the evaluation of machine learning algorithms. Pattern Recognition 30: 1145-1159, 1997.

BUCKLEY JJ, ESLAMI E: An Introduction to Fuzzy Logic and Fuzzy Sets. Springer, New York, 2002, 285 pp.

CAMM AJ, FEI L: Risk stratification following myocardial infarction: Heart rate variability and other risk factors. In: Heart Rate Variability. M MALIK, AJ CAMM (eds), Armonk, New York, 1995, pp 369-392.

FRANK E, HARRELL J: Regression Modeling Strategies. Springer, New York, 2001, 568 pp.

GREISER KH, KLUTTIG A, SCHUMANN B, SWENNE CA, KORS JA, KUSS O, HAERTING J, SCHMIDT H, THIERY J, WERDAN K: Cardiovascular diseases, risk factors and short-term heart rate variability in an elderly general population: the CARLA study 2002-2006. Eur J Epidemiol 24: 123-142, 2009.

HONZÍK P, HRABEC J, LÁBROVÁ R, SEMRÁD B, HONZÍKOVÁ N: Fuzzification, weight and summation of risk factors in a patient improves the prediction of risk for cardiac death. Scripta Medica 76: 141-148, 2003.

HONZÍKOVÁ N, FIŠER B, HONZÍK J: Noninvasive determination of baroreflex sensitivity in man by means of spectral analysis. Physiol Res 41: 31-37, 1992.

HONZÍKOVÁ N, SEMRÁD B, FIŠER B, LÁBROVÁ R: Baroreflex sensitivity determined by spectral method and heart rate variability, and two-years mortality in patients after myocardial infarction. Physiol Res 49: 643-650, 2000a.

HONZÍKOVÁ N, FIŠER B, SEMRÁD B: Critical value of baroreflex sensitivity determined by spectral analysis in risk stratification after myocardial infarction. PACE 23: 1965-1967, $2000 \mathrm{~b}$.

HONZÍKOVÁ N, SEMRÁD B, LÁBROVÁ R, HRABEC J, HONZÍK P: Risk stratification by fuzzy and weighted methods. Physiol Res 52: 28P, 2003.

KRONTORÁDOVÁ K, HONZÍKOVÁ N, FIŠER B, NOVÁKOVÁ Z, ZÁVODNÁ E, HRSTKOVÁ H, HONZÍK P: Overweight and decreased baroreflex sensitivity as independent risk factors for hypertension in children, adolescents, and young adults. Physiol Res 57: 385-391, 2008.

LA ROVERE MT, BIGGER JT Jr, MARCUS FI, MORTARA A, SCHWARTZ PJ: Baroreflex sensitivity and heart-rate variability in prediction of total cardiac mortality after myocardial infarction. ATRAMI (Autonomic tone and reflexes after myocardial infarction) Investigators. Lancet 351: 478-484, 1998.

MANGONI ED, ADINOLFI LE, TRIPODI MF, ANDRENA A, GAMBARDELLA M, RAGONE E, PRECONE DF, UTILI R, RUGIERO G: Risk factors for „major“ embolic events in hospitalized patients with infective endokarditis. Am Heart J 146: 311-316, 2003.

MORTARA A, SPECCHIA G, LA ROVERE MT, BIGGER JT Jr, MARCUS FI, CAMM JA, HOHNLOSER SH, NOHARA R, SCHWARTZ PJ: Patency of infarct-related artery. Effect of restoration of anterograde flow on vagal reflexes. ATRAMI (Automatic Tone and Reflexes After Myocardial Infarction) Investigators. Circulation 93: 1114-1122, 1996.

MOSS AJ, HALL WJ, CANNOM DS, DAUBERT JP, HIGGINSS SL, KLEIN H, LEVINE JH, SAKSENA S, WALDO AL, WILBER D, BROWN MW, HEO M: Improved survival with an implanted defibrillator in patients with coronary disease at high risk for ventricular arrhythmia. Multicenter Autonomic Defibrillator Implantation Trial Investigators (comment). N Engl J Med 335: 1933-1940, 1996. 
ZAVODNA E, HONZIKOVA N, HRSTKOVA H, NOVAKOVA Z, MOUDR J, JIRA M, FISER B: Can we detect the development of baroreflex sensitivity in humans between 11 and 20 years of age? Can J Physiol Pharmacol 84: 1275-1283, 2006. 